\title{
Performance evaluation of the IEEE 802.11n random topology WLAN with QoS application
}

\author{
Ziyad Khalaf Farej, Mustafa Mohammad Jasim \\ Department of Computer Engineering, Northern Technical University, Iraq
}

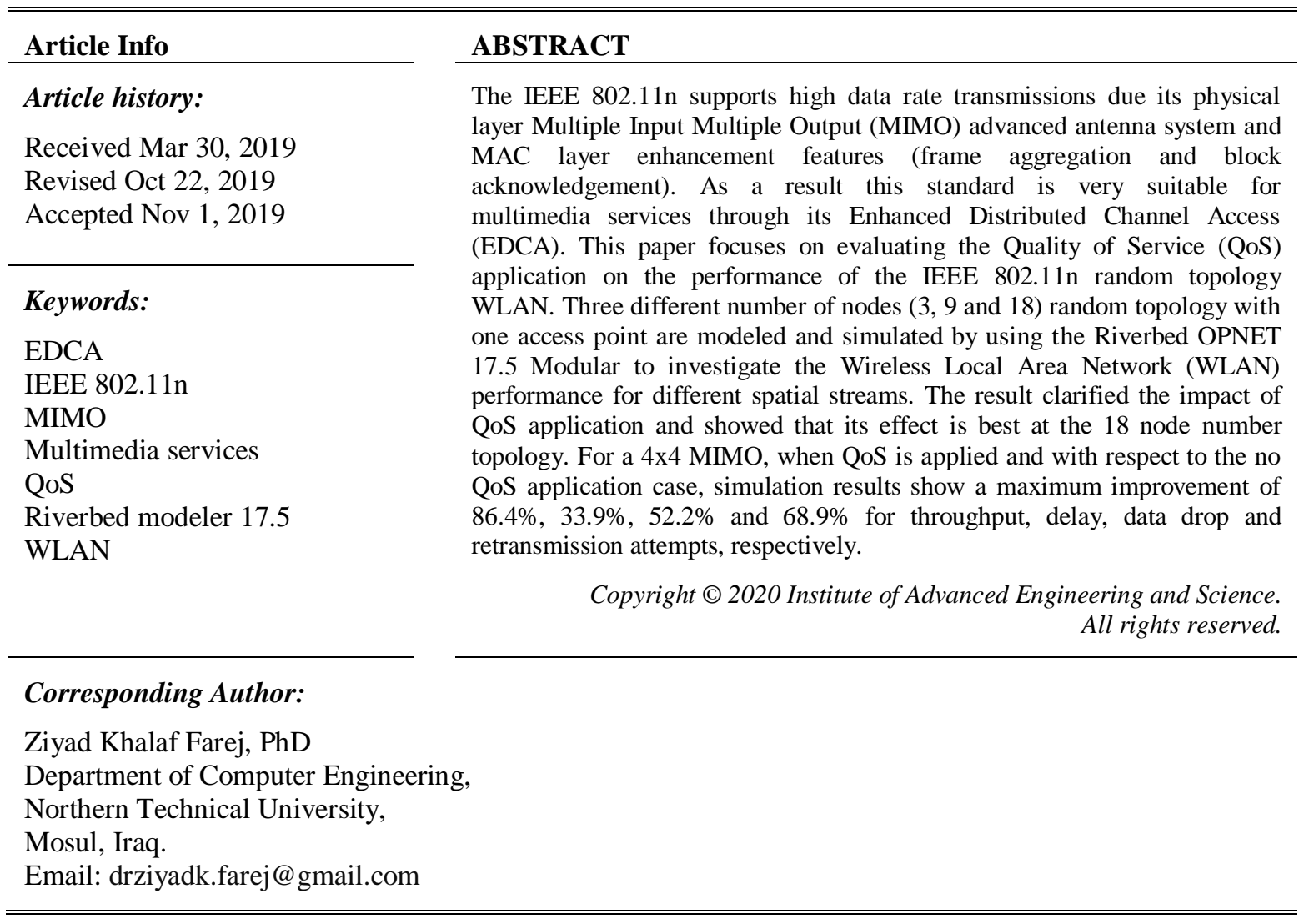

\section{INTRODUCTION}

Researches on wireless networks aim for feasible and cheap broadband internet access, as well as investigation on Quality of Service (QoS) for critical time services systems (such as healthcare and telemedicine, emergency and disaster networking systems) have recently attracted considerable attention. Wireless multi-hop networks, such as wireless mesh networks (WMNs) random topology, are considered an ideal networks field for such services [1] thus; multi-hop wireless networks become necessary for users. However due to the limitation of the transmission range of wireless network interfaces, multiple hops are needed to extend range and exchange data between stations in the network and the IEEE 802.11 standard is widely applied in multi-hop wireless networks $[2,3]$. The problem with these networks is the need for high channel capacity and throughput to satisfy the requirement of advanced wireless workstations for high data rate and QoS application. This problem is addressed through the IEEE 802.11n standard improvements. These improvements include the physical layer Multiple Input Multiple Output (MIMO) smart antenna system and the necessary MAC layer enhancement such as frame aggregation and Block Acknowledgement (BA) [4]. The frame aggregation mechanism includes two levels (MAC Service Data Unit (MSDU) and MAC Protocol Data Unit (MPDU)) of aggregations. All the above cause the standard data rate and spectral efficiency to be increased by sending more data frames in a single transmission. Also BA improves the MAC efficiency to resolves the drawback of large aggregation without the need to acknowledge every received unicast frame, so it is obviously suitable for unicast and delay sensitive or real time applications where 
retransmission is critical, as a result throughput can be significantly increased $[5,6]$. The random topology is considered to deploy multi-hop and extend network range. As a result the performance of the network is degraded. In this paper the QoS, which is an IEEE 802.11n feature, is applied and its effects on the Wireless LAN performance is investigated. In comparison with its previous counterpart the IEEE 802.11n has better performance, compatible with previous WLAN standards and supports QoS protocol of the IEEE 802.11e which grants differentiated services for voice, video and data [7, 8].The changes at the physical and MAC layers of the IEEE 802.11n standard enhance the previous IEEE 802.11 standards and the peak data rate of this standard may reach around 300 or $600 \mathrm{Mbps}$ when working on the $2.4 \mathrm{or} 5 \mathrm{GHz}$ band with 20 or $40 \mathrm{MHz}$ channel bandwidth respectively [5, 6, 8, 9].

\section{RELATED WORK}

The performance of the WLAN based on the IEEE 802.11n standard is better when using two level frame aggregations at the MAC layer and MIMO-OFDM at physical layer compared to the no frame aggregation case, reference [10] shows improvement in the performance with values of $37.4 \%$ and $13.7 \%$ in terms of throughput and end to end delay respectively. The authors in [11] proposed a routing protocol for wireless body area network to transmit data with low energy consumption value and high network life time, moreover they presented a multi-hop random topology and performance evaluation using simulations approach. In [12], the authors proposed a scheme which is based on IEEE 802.11 standard for multi-hop wireless networks with Transmission Opportunity (TXOP) to support QoS for wireless networks. Their scheme assigns the variable length of TXOPs to different traffics based on precise channel condition prediction. They concluded that their scheme improved the network reliability (by allowing more failure recovery times), reduced delay and increased throughput. The authors in [3] presented an approximated analytical model for the throughput and delay performance for multi-hop ad hoc networks, they show an obvious improvement on the 8 nodes random topology performance of the IEEE 802.11a standard with enhanced DCF, their improvement values with QoS applications are 7.14\% and 40\% for throughput and delay respectively.

The authors in [13] concentrated on the IEEE 802. 11 Mac layer parameters and used the OPNET network simulation tool to show that the WLAN performance can be improved by fine tuning parameters such as fragmentation threshold, Request to Send (RTS) thresholds and buffer size. The authors in [14] summarized the Modified Binary Particle Swarm Optimization (MBPSO) algorithm to improve the performance of the wireless network random topology. With the used optimization function and the distance between nodes taken into consideration, their result show that the maximum improvement is $8.33 \%, 5.79 \%$ and $17.83 \%$ for end to end delay, throughput and packet delivery ratio respectively. Many other attempts have been made by references $[15,16]$ to provide more simple analytical models to compute throughput and delay. Their analysis without hidden node problem show that interference among nodes can be reduced to maximize throughput by using the smallest transmission range and the achievable per node throughput is $11 \%$ of the channel capacity. The authors in [17] have considered the effect of QoS application on the IEEE 802.11n based uniform (or single hop) topology performance. For 18-node uniform topology with $4 \times 4$ spatial streams, their results show that when QoS is applied the maximum improvement values are $91.6 \%$ and $34.8 \%$ for throughput and delay respectively. This paper investigates the IEEE $802.11 \mathrm{n}$ random topology performance and the effect of QoS application. In comparison with [17] which is part of our MSc. research, the high number of nodes random topology shows higher throughput performance. However when QoS is applied the improvement is lower than that of the uniform topology. The reason for that is the bottleneck or access point congestion of uniform topology is distributed over the multiple hops of the available different routes for random topology.

\section{IEEE 802.11n AMENDMENTS AND QoS APPLICATION}

IEEE 802.11n has higher throughput improvements and better spectral efficiency due to the improvement of the physical layer (MIMO) antenna communication system and the necessary MAC layer enhancement such as the two level (A-MSDU with maximum size is 7935 bytes and A-MPDU with maximum size is 65535 bytes) frame aggregation mechanism and block acknowledgement that resolves the drawback of large aggregation by retransmitting only erroneous frames as shown in Figure $1[5,6,18,19]$.

QoS technology is applied to manage and investigate the performance of the high data traffic load for high number of nodes network model and also in order to perfectly evaluate the performance (throughputs, packet loss and reduce latency) of the network [20-22]. Through the data flow management process with priorities for the different services, a certain level of data flow is ensured and as a result 
performance is improved. The QoS is implemented through the application of the MAC layer Hybrid Coordination Function (HCF) with Enhanced Distributed Channel Access (EDCA) mechanism as shown in Figure 2(a) [23]. According to this necessary function, the parameters on which the QoS is prioritized are the minimum size Contention Window (CWmin) and maximum size contention window (CWmax) techniques which count on providing the required Arbitrary Inter Frame Space (AIFS) and Transmission Opportunity (TXOP). It shows that the back-off of the highest priority access category (AC) is assigned the lowest value of AIFS; the TXOP is a bounded time interval during which a node can send as many frames as possible. In these scenarios when QoS is applied, the QoS parameters are listed in Table 1 and the RTS threshold was used and determined by a frame length of 512 bytes. In our performance analysis the scheduled service queues are considered as Voice, Video and Best effort (FTP) as shown in Figure 2(b).

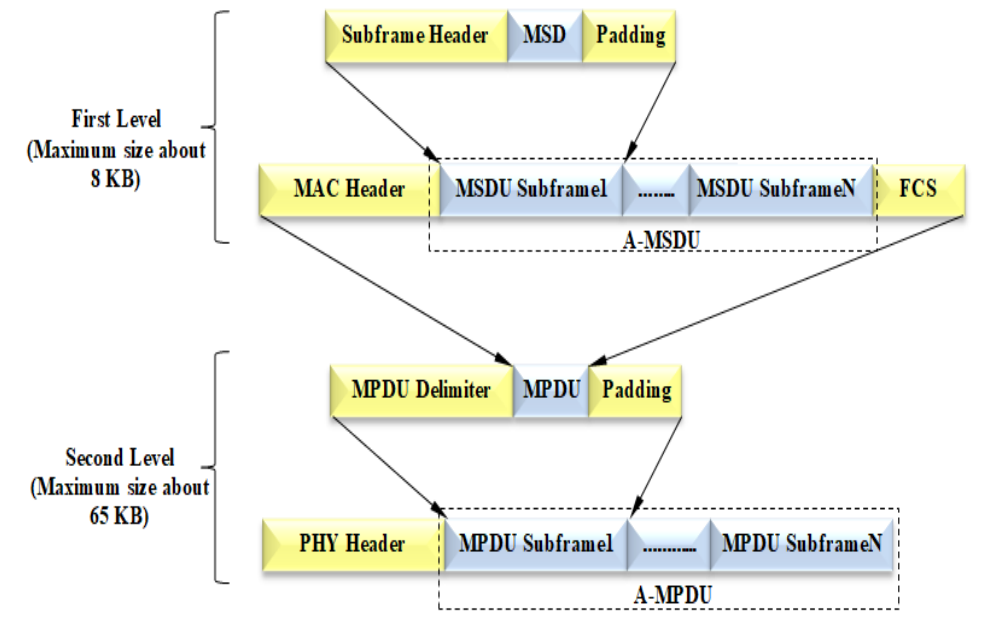

(a)

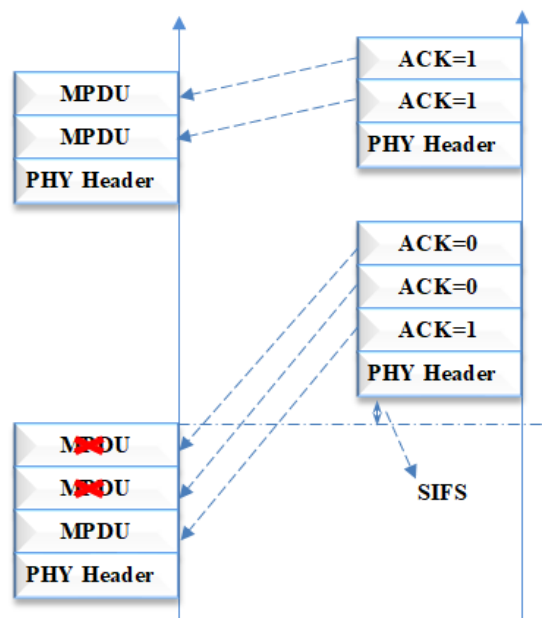

(b)

Figure 1. (a) Two level frame aggregation; (b) Block acknowledgement in IEEE 802.11n standard

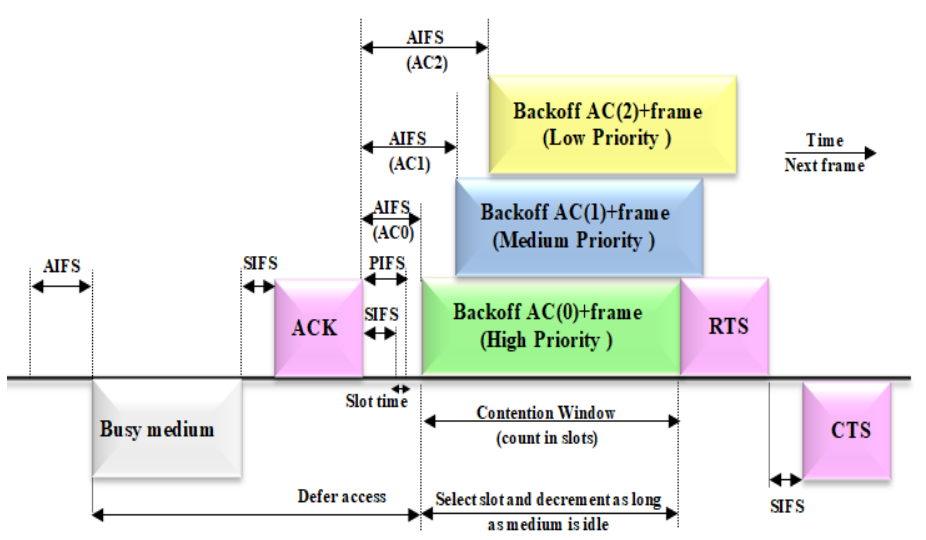

(a)

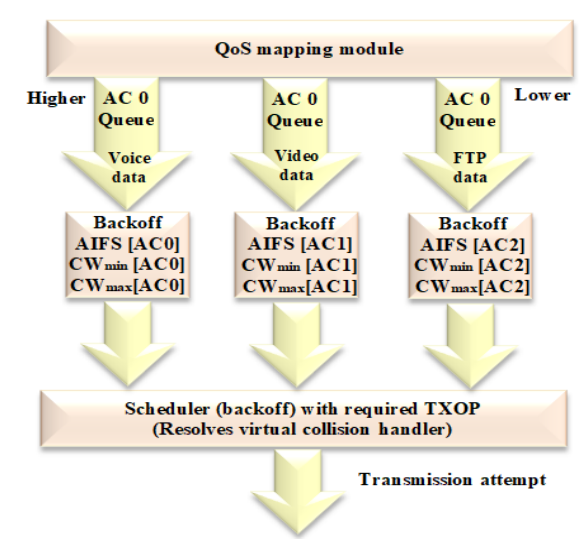

(b)

Figure 2. (a) Timing diagram representation of EDCA; (b) QoS mapping module

Table 1. EDCA (QoS) prioritized parameters

\begin{tabular}{cccc}
\hline Parameters & \multicolumn{3}{c}{ Values } \\
& Voice & Video & Best effort \\
\hline Max TXOP (ms) & 6.016 & 6.016 & 3.264 \\
AIFSN(back-off) (slot) & 2 & 2 & 3 \\
CWmin (slot) & 3 & 7 & 15 \\
CWmax (slot) & 7 & 15 & 1023 \\
\hline
\end{tabular}




\section{THEORETICAL PERFORMANCE OF MULTI-HOP WIRELESS NETWORKS}

This type of Networks offers communications capability to multiple users with a fixed infrastructure. Each node in a wireless multi-hop network functions as both a host and a router, and the nodes in this network forward data to the destination according to the Ad-hoc routing protocol. The network packets can traverse multiple intermediate nodes from the source to the destination and each forwarding step is called a hop. A simple uniform multi-hop wireless network, which forms the basis of the WMN random topology, is shown in Figure 3 [24, 25].

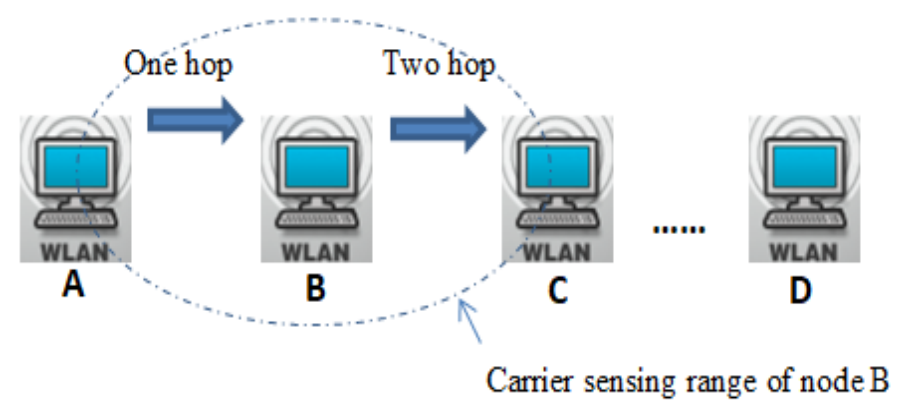

Figure 3. Multi-hop wireless ad-hoc networks

A simple mathematical model that provides the upper bond throughput and delay is given by [26]:

$$
\begin{aligned}
& \text { Max Data Rate (bits/sec. })=\frac{\mathrm{DS} * \mathrm{SS} * \text { Bits Per Symbol } * \mathrm{CR}}{\text { Symbol Duration }} \\
& \text { Delay }_{\text {sec }}=\frac{\mathrm{L}_{\text {max }}}{\text { Max Data Rate }}
\end{aligned}
$$

Where:

DS = Data Subcarriers (equal to 52 for $20 \mathrm{MHz}$ ),

$\mathrm{SS}=$ Spatial Stream is variable from one up to four,

Bits per Symbol are 6 for 64-QAM,

$\mathrm{CR}=$ Code Rate is $5 / 6$,

Symbol Duration equal 3.6 $\mu$ s include guard interval of $400 \mathrm{~ns}$,

$\mathrm{L}_{\max }$ is the A-MPDU length which is 65535 bytes and Table 2 shows the maximum theoretical throughput and delay for different spatial stream.

Table 2. Shows the maximum theoretical throughput and overall delay values of the $802.11 \mathrm{n}$ standard

\begin{tabular}{ccccc}
\hline Spatial stream & $1 \times 1$ & $2 \times 2$ & $3 \times 3$ & $4 \times 4$ \\
\hline Throughput (Mbps) & 72.2 & 144.4 & 216.6 & 288.8 \\
Delay (Sec.) & 0.00726 & 0.00363 & 0.00241 & 0.00181 \\
\hline
\end{tabular}

\section{RANDOM TOPOLOGY WIRELESS NETWORKS MODELING AND ASSUMPTIONS}

To test the real network behavior as the range extended with high load, three randomly distributed node topologies with different (3, 9 and 18) number of nodes are considered as shown in Figure 4. These topologies are modeled and simulated using the OPNET (Riverbed) modeler version 17.5. The nodes in these topologies transmit multimedia services (for monitoring and diagnostic) such as audio, video, and urgent images to the monitoring center (destination). Based on the QoS parameters which are introduced in Table 1 and under the assumption that no hidden terminal problem exists, these topologies are considered (with an ADOV routing protocol) to investigate the performance of the IEEE 802.11n based WLAN under the following simulation set up parameters as shown in Table 3. 

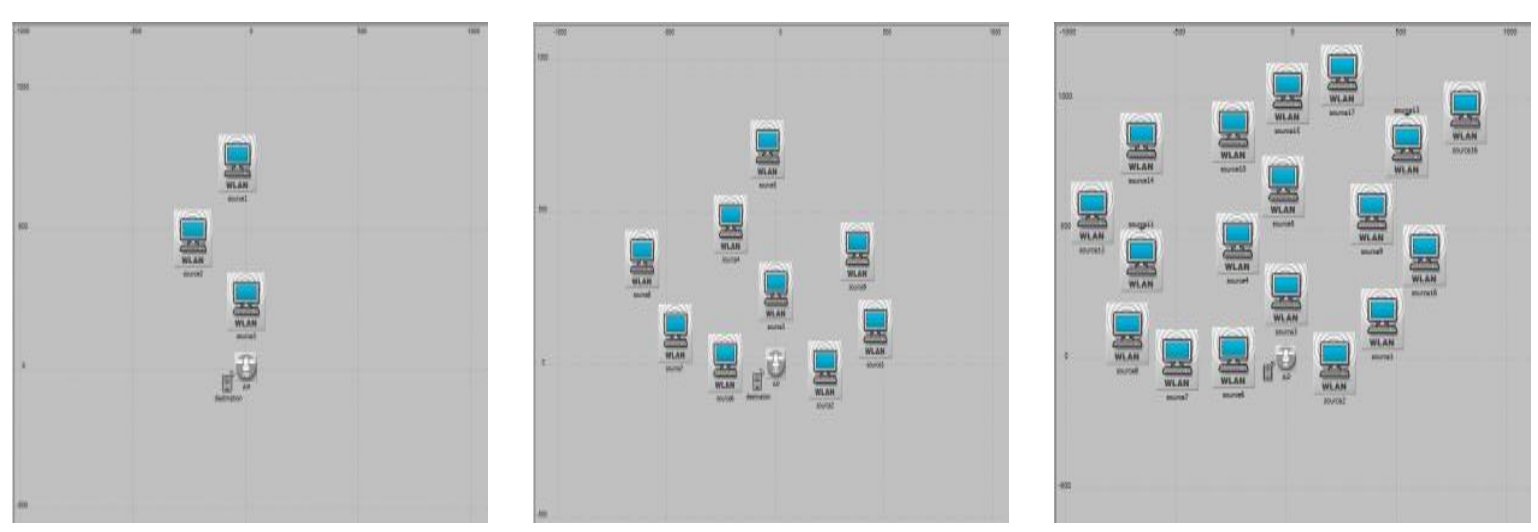

Figure 4. 3, 9 and 18-node random topology

Table 3. WLAN based $802.11 \mathrm{n}$ simulation parameters

\begin{tabular}{ll}
\hline Parameters & Values \\
\hline Randomly distributed area & $1000 \times 1000 \mathrm{~m}$ \\
Buffer size (bits) & 1024000 \\
Data rate (Mbps) & 288 \\
packet size (bytes) & 2048 \\
Standard slot time ( $\mu \mathrm{sec})$ & 9 \\
Voice type & $\mathrm{G} .711$ (encoder scheme) \\
Video type & 30 frame/sec- 352x240 pixel \\
Files information (bytes) & high load \\
Transmit power (Watt) & 0.1 \\
Packets reception power threshold (dbm) & -95 \\
RTS Threshold (bytes) & $(512 / \mathrm{None})$ and CTS to-self Option is Enabled \\
Frame aggregation A-MSDU (bytes) & 7935 \\
Frame aggregation A-MPDU (bytes) & 65535 \\
physical characteristics & $802.11 \mathrm{n}, 2.4$ GHzwith channel BW 20 MHz \\
Block Acknowledgement capability & Supported \\
Simulation time (min) & 1 \\
\hline
\end{tabular}

\section{IEEE 802.11n WLAN WITH RANDOM TOPOLOGY RESULT ANALYSIS}

The performance of the networks with random topology has been investigated in terms of (Throughput, Delay, Packets Drops and Retransmissions attempts). The simulation processes are carried out under the network assumptions and simulation set up parameters that have been introduced in Table 3 and Table 1 previously. Different numbers (1,2,3 and 4) of spatial streams are considered in the process of investigating the performance of the IEEE 802.11n WLAN efficiency.

\subsection{Throughput}

As a matter of fact and for the different spatial streams $1 \times 1,2 \times 2,3 \times 3$ and $4 \times 4$ configuration, the average throughput gradually increases as the network is loaded with data. From Figure 5 (a-d), it can be noted that the maximum throughput value decreases as the numbers of nodes increased from 3 nodes to 18 nodes due to more collision and packets drop when high number of packets are injected to the coordinator. But when QoS is implemented, the throughput is improved and that is obvious specially at high number of nodes and for different antennas configuration; the reason for that is QoS provides priorities to the scheduled services and organizes data flowing. Without and with QoS and for a 1x1 antenna system, the results show that the maximum throughput values that can be acquired are $(57.3,40.8,30.5 \mathrm{Mbps})$ and (61.4, 54.6, $53.7 \mathrm{Mbps})$ for 3, 9 and 18 nodes scenarios respectively. So when QoS is applied the improvement values are $6.9 \%, 33.8 \%$ and $76 \%$ for 3,9 and 18 nodes scenarios respectively. Although the maximum throughput value is at the lowest (3) node number topology, however the highest improvement is obtained at the highest (18) node number topology; the reason for that is the effect of QoS becomes more obvious when congestion with number of hops are increased. So QoS application is required to improve network performance. 


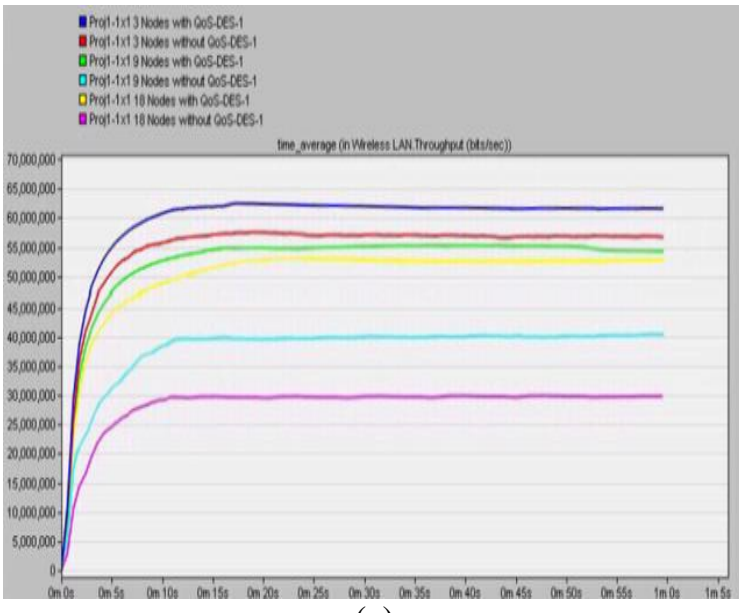

(a)

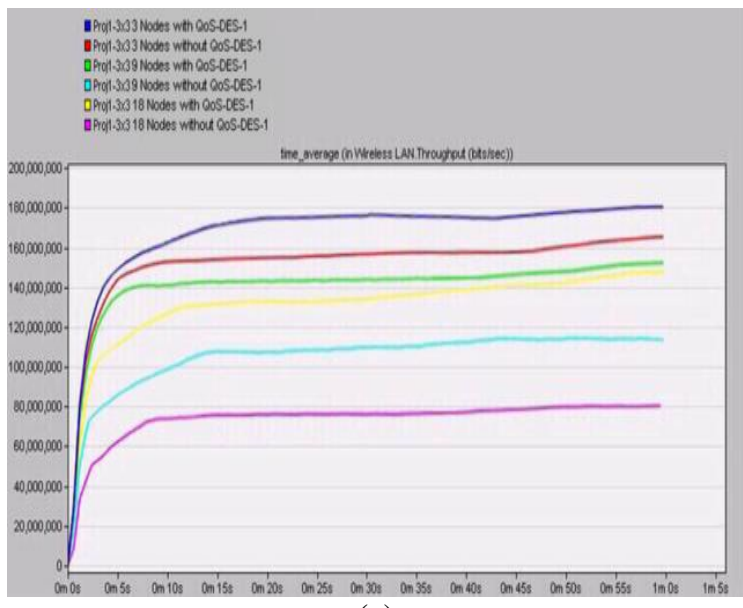

(c)

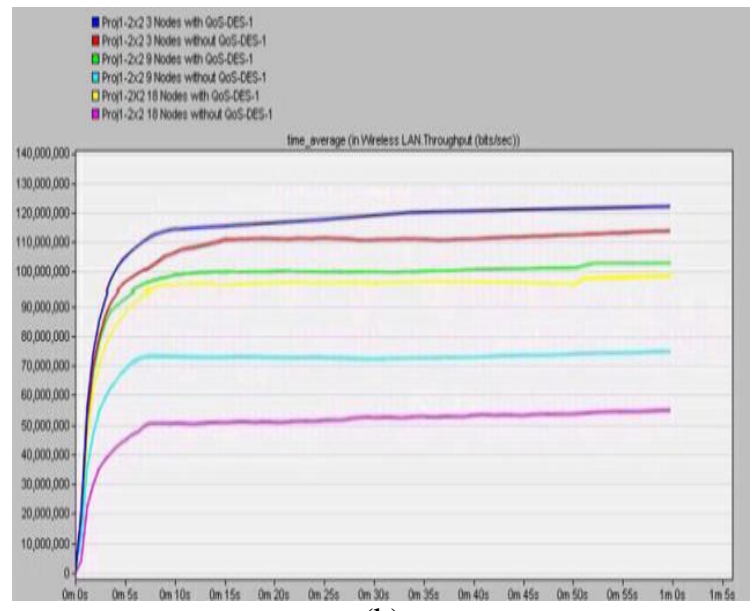

(b)

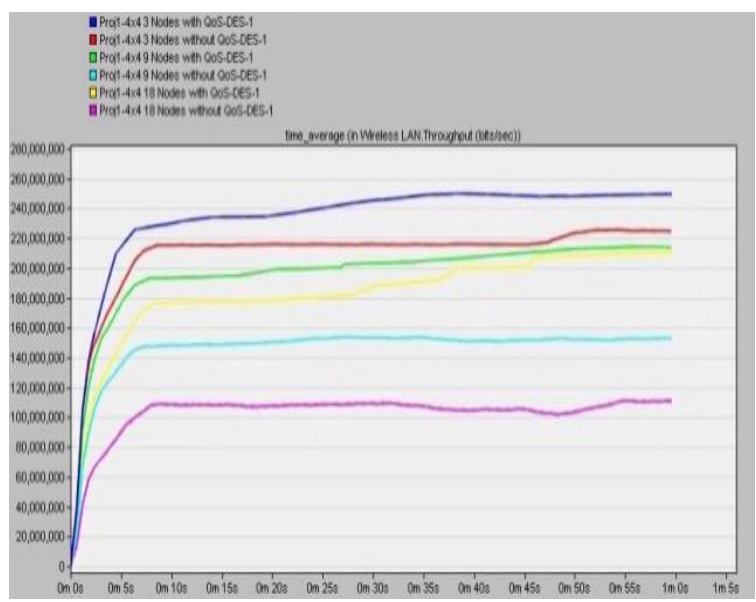

(d)

Figure 5(a, b, c and d). Shows throughput performance with and without QoS application for $(1 \times 1,2 \times 2,3 \times 3$ and $4 \times 4)$ antenna systems respectively

\subsection{Delay}

Figure 6 (a, b, c and d) shows delays for different number of spatial streams also with and without QoS application. Through the increase of the data load (number of nodes) of the network, the delay is increased as well and that leads to more collision and retransmission attempts. This lateness ensures handing of the data when the WLAN is crowded. Without QoS and for 1x1 antenna system the average delay has maximum steady state values of $0.0763,0.1459$ and 0.278 seconds for 3,9 and 18 nodes number scenarios respectively. However when QoS is applied and data is scheduled, the latency improvement (reduction percentage in delay) for high number of nodes module is the best and it has the values of $8.5 \%$, $12.9 \%$ and $27.6 \%$ for nodes number of 3,9 and 18 values respectively, where the maximum delay values are $0.0698,0.0127$ and 0.201 seconds respectively as well.

\subsection{Data drop}

Figure 7(a-d) illustrates the data packets drop for different number of nodes (3,9 and 18) with spatial streams (1, 2, 3 and 4). In general, data packets drop is growing up over time as the network is loaded with data. Figure 7(a) shows that the data drop increases as the number of nodes increases and (for 1x1 spatial stream) the maximum average packet drop is $(61,69.8,78.6)$ and $(76.9,99.7,137.4)$ with and without QoS respectively. The increase in the dropped data packets is due to the collisions which are as well increases as the number nodes are increased, however for the mesh (or random) topology, the collision domain is distributed over all the nodes along the routing paths and the collisions are not concentrated at the coordinator as in uniform (single hope) topology where the network suffering high dropping packets when it is highly loaded. 


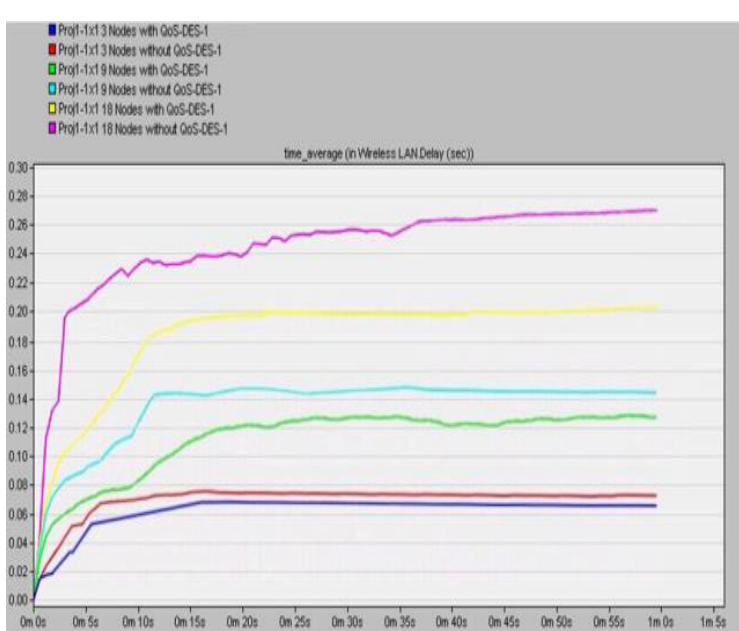

(a)

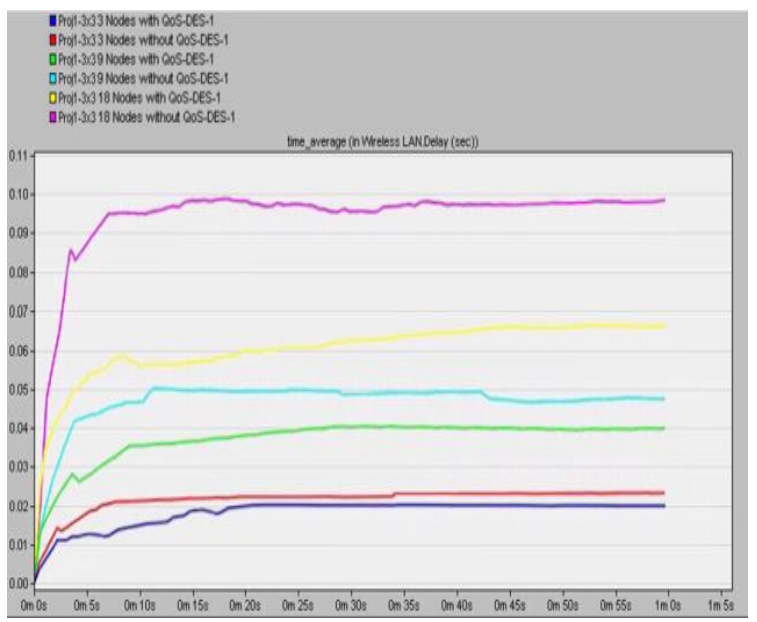

(c)

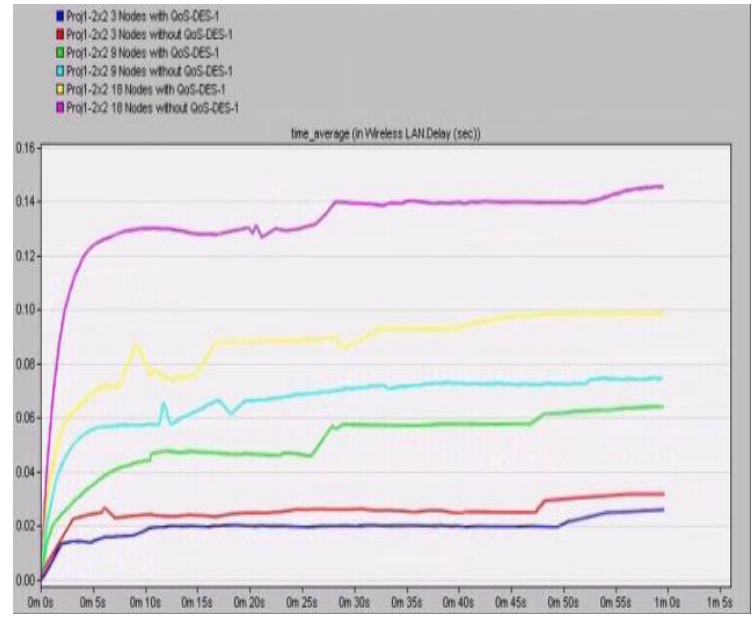

(b)

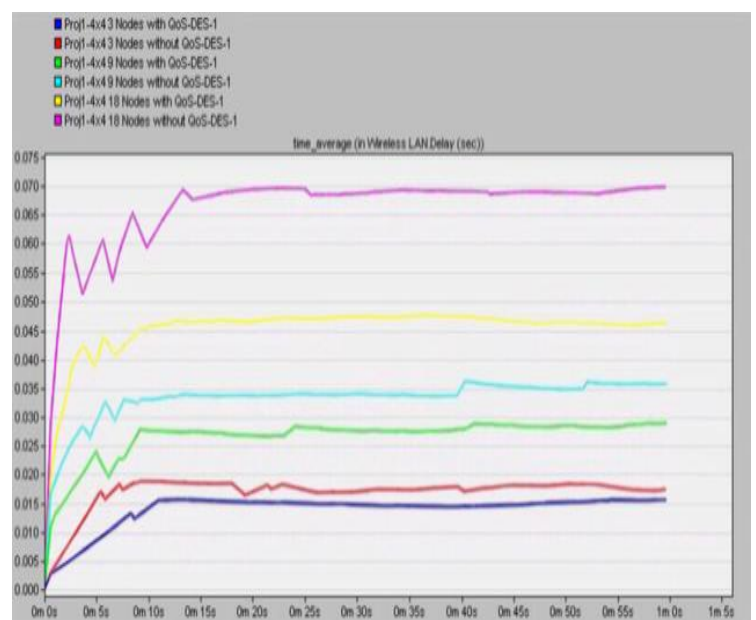

(d)

Figure 6(a, b, c and d). Shows delay performance with and without QoS application for $(1 \mathrm{x} 1,2 \mathrm{x} 2,3 \times 3$ and $4 \times 4)$ antenna systems respectively

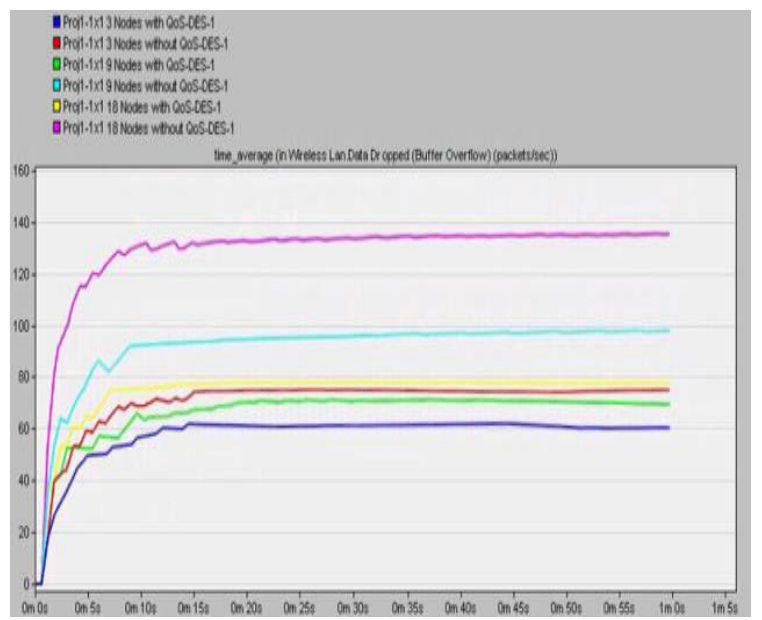

(a)

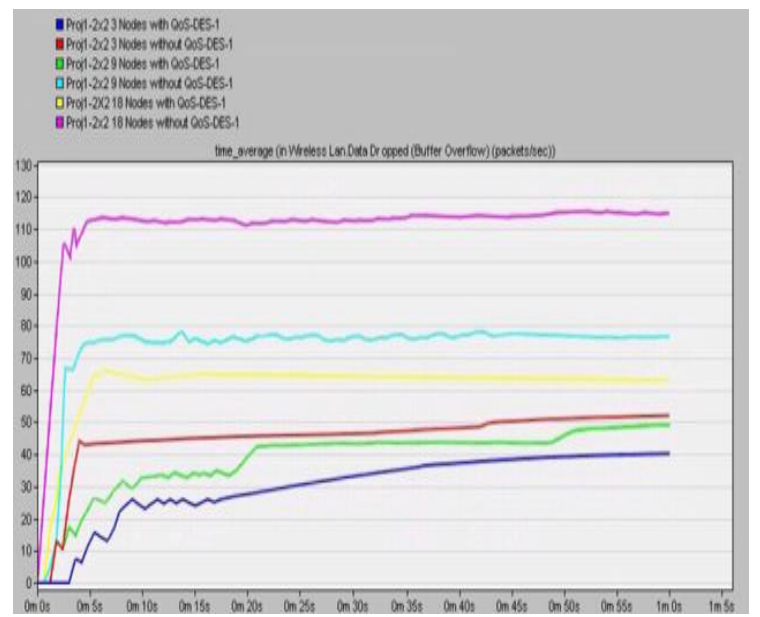

(b)

Figure 7. (a, b) Shows data drop performance with and without QoS application for $(1 \times 1,2 \times 2,3 \times 3$ and $4 \times 4)$ antenna systems respectively 


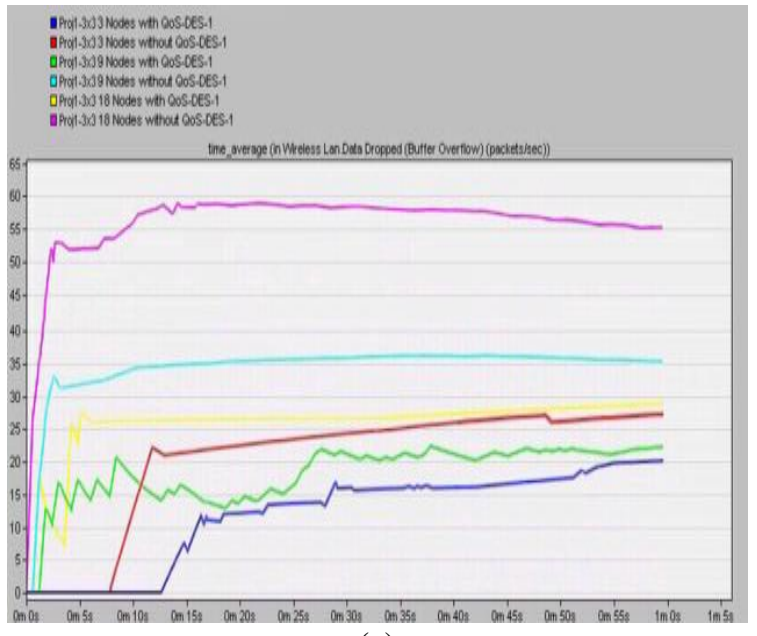

(c)

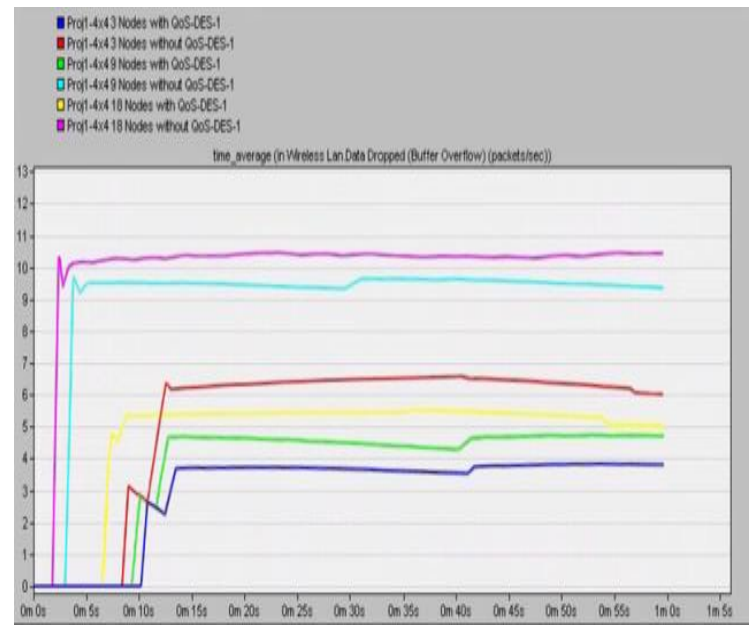

(d)

Figure 7. (c, d) Shows data drop performance with and without QoS application for $(1 \times 1,2 \times 2,3 \times 3$ and $4 \times 4)$ antenna systems respectively

\subsection{Retransmission attempt}

Figure $8(\mathrm{a}, \mathrm{b}, \mathrm{c}$ and $\mathrm{d})$ shows the result of the retransmission attempts which are for 18 nodes are fairly more than that for 9 and 3 node topologies due to the load increase of the WLAN, congestion and data packet dropping. Retransmission attempts decreases and data packets drop decreases as the number of spatial streams increases due to channel capacity enhancement. However in comparison with no QoS case, when QoS is considered, the retransmission attempts are increased by the QoS data scheduler to reduce the packets drop chances. For a $4 \times 4$ spatial stream with and without QoS application, Figure 8(d) shows that the maximum average retransmission attempt values are $(0.495,2,3.92)$ and $(0.371,1.47,2.32)$ for 3,9 and 18 number of nodes respectively, where it is clear that the best improvement (highest retransmission attempt i.e. least packet drop) of $68.9 \%$ is acquired at 18 node number scenario. The performances of the throughput, delay, packets drops and retransmission attempts with their improvements, when QoS is applied, are summarized in Tables 4, 5, 6 and 7 respectively.

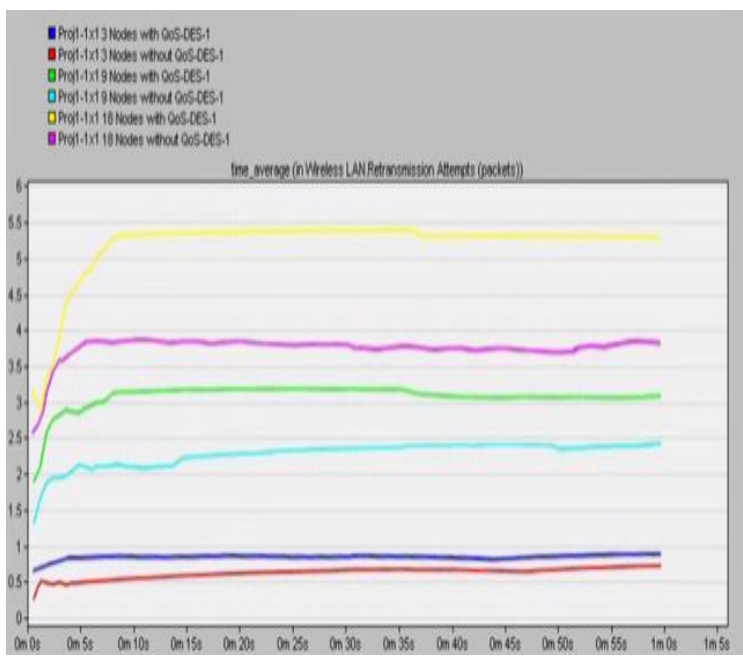

(a)

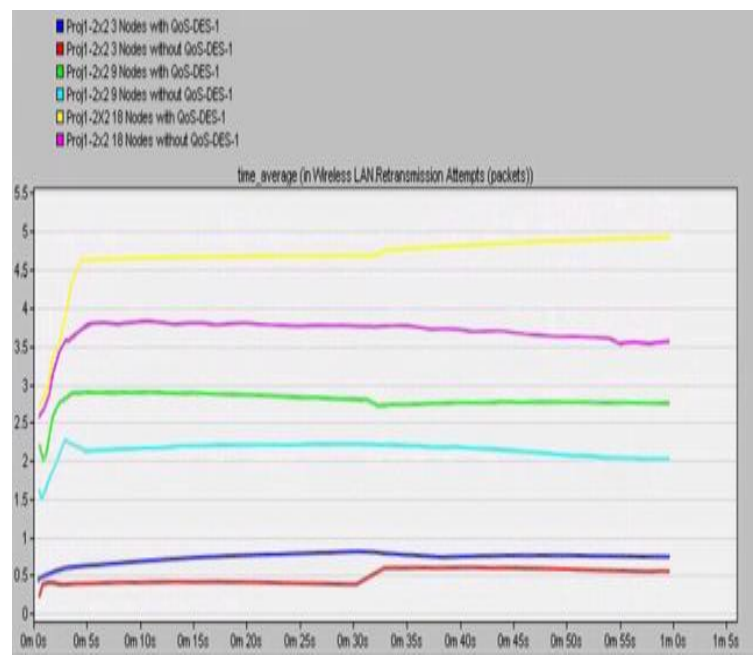

(b)

Figure 8. (a, b) Shows retransmission attempt performance with and without QoS application for $(1 \times 1,2 \times 2,3 \times 3$ and $4 \times 4)$ antenna systems respectively 


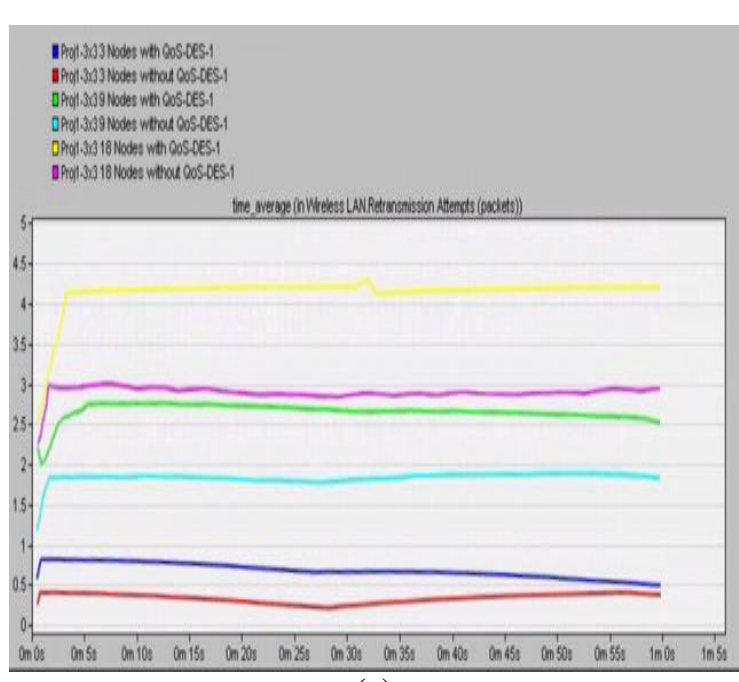

(c)

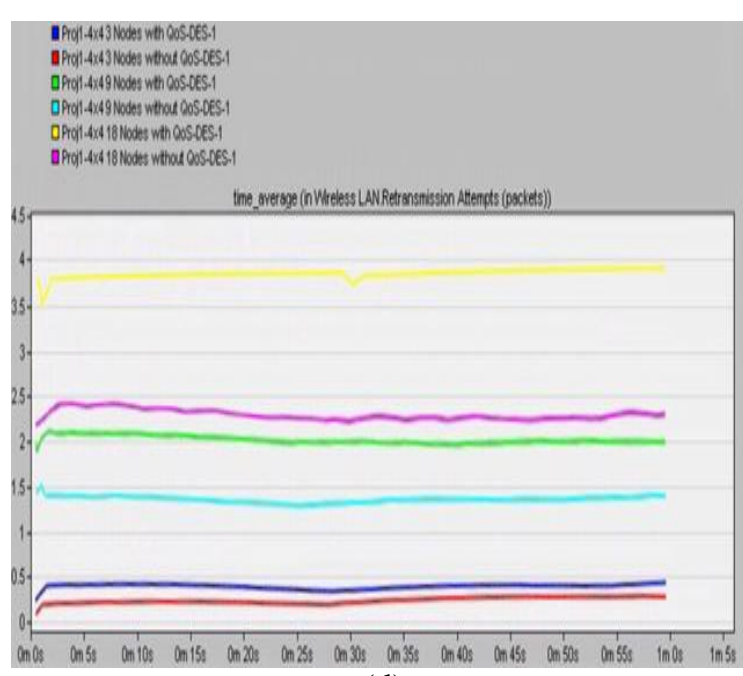

(d)

Figure 8. (c, d) Shows retransmission attempt performance with and without QoS application for $(1 \times 1,2 \times 2,3 \times 3$ and $4 \times 4)$ antenna systems respectively

Table 4. Summarizing throughput performance

\begin{tabular}{cccccccccc}
\hline Spatial & & \multicolumn{3}{c}{ 3 Nodes } & \multicolumn{5}{c}{ 9 Nodes } \\
Streams & Without QoS & With QoS & \% Improve & Without QoS & With QoS & \% Improve & Without QoS & With QoS & \% Improve \\
\hline 1x1 & 57.383 & 61.4 & 6.9 & 40.8 & 54.6 & 33.8 & 30.5 & 53.7 & 76 \\
$2 \times 2$ & 113.4 & 112 & 7.5 & 76.402 & 102.5 & 34.1 & 54.9 & 99.8 & 81.7 \\
$3 \times 3$ & 166.2 & 180.9 & 8.8 & 113.9 & 154.7 & 35.8 & 80.7 & 148.1 & 83.5 \\
$4 \times 4$ & 224.9 & 248 & 10.2 & 156 & 215 & 37.8 & 111.04 & 210.01 & 86.4 \\
\hline
\end{tabular}

Table 5. Summarizing delay performance

\begin{tabular}{cccccccccc}
\hline Spatial & & 3 Nodes & & \multicolumn{4}{c}{ 9 Nodes } \\
Streams & Without QoS & With QoS & \% Improve & Without QoS & With QoS & \% Improve & Without QoS & With QoS & \% Improve \\
\hline 1x1 & 0.07631 & 0.0698 & 8.5 & 0.1459 & 0.127 & 12.9 & 0.2780 & 0.201 & 27.6 \\
$2 \times 2$ & 0.0320 & 0.0287 & 10.5 & 0.0751 & 0.064 & 14.7 & 0.1434 & 0.0997 & 30.4 \\
$3 \times 3$ & 0.0231 & 0.0205 & 11.2 & 0.0489 & 0.0401 & 17.9 & 0.981 & 0.067 & 31.7 \\
$4 \times 4$ & 0.0174 & 0.0151 & 13.2 & 0.0366 & 0.0298 & 18.3 & 0.0709 & 0.0468 & 33.9 \\
\hline
\end{tabular}

Table 6. Summarizing data drop performance

\begin{tabular}{cccccccccc}
\hline Spatial & & 3 Nodes & \multicolumn{5}{c}{ 9 Nodes } & \multicolumn{3}{c}{18 Nodes } \\
Streams & Without QoS & With QoS & \% Improve & Without QoS & With QoS & \% Improve & Without QoS & With QoS & \% Improve \\
\hline $1 \times 1$ & 76.985 & 61.093 & 20.6 & 99.78 & 69.8 & 30 & 137.4 & 78.6 & 42.5 \\
$2 \times 2$ & 51.8 & 40.7 & 21.4 & 77.3 & 49.1 & 36.4 & 116.8 & 63.5 & 45.6 \\
$3 \times 3$ & 27.895 & 20.4 & 26.8 & 35.817 & 22.401 & 37. & 55.670 & 28.7 & 48.3 \\
$4 \times 4$ & 6.009 & 3.978 & 33.7 & 9.279 & 4.8 & 48.2 & 10.522 & 5.019 & 52.2 \\
\hline
\end{tabular}

Table 7. Summarizing retransmission attempt performance

\begin{tabular}{|c|c|c|c|c|c|c|c|c|c|}
\hline \multirow{2}{*}{$\begin{array}{l}\text { Spatial } \\
\text { Streams }\end{array}$} & \multicolumn{3}{|c|}{3 Nodes } & \multicolumn{3}{|c|}{9 Nodes } & \multicolumn{3}{|c|}{18 Nodes } \\
\hline & Without QoS & With QoS & $\%$ Improve & Without QoS & With QoS & $\%$ Improve & Without QoS & With QoS & \% Improve \\
\hline $1 \times 1$ & 0.711 & 0.905 & 27.2 & 2.401 & 3.116 & 29.7 & 3.896 & 5.310 & 36.2 \\
\hline $2 \times 2$ & 0.560 & 0.729 & 30.1 & 2.04 & 2.787 & 33.3 & 3.6 & 4.990 & 38.6 \\
\hline $3 \times 3$ & 0.406 & 0.534 & 31.5 & 1.821 & 2.534 & 39.1 & 2.99 & 4.252 & 45.5 \\
\hline $4 \times 4$ & 0.371 & 0.495 & 33.4 & 1.47 & 2 & 42.8 & 2.32 & 3.92 & 68.9 \\
\hline
\end{tabular}

\section{CONCLUSION}

This paper presents an investigation and performance evaluation of the IEEE 802.11n random topology WLAN for multimedia services. The optimal configuration of the standard MAC layer features with the suitable physical layer spatial stream clarified the impact of the standard on the network performance. Although throughput value decreases, the effect of QoS on improving the WLAN 
network efficiency increases and becomes more obvious as the number of nodes increases. Without and with QoS and for a 4x4 MIMO, simulation results show that the maximum average throughput can be acquired at the lowest node number topology and its values are $(224.9,156,111.04 \mathrm{Mbps})$ and $(248,215,210.01 \mathrm{Mbps})$ for 3, 9 and 18 node number topology respectively. The simulation result, which are within the upper bond theoretical results also reflects the efficiency and feasibility of the standard for high load networks specially when services are scheduled through the QoS process according to its EDCA mechanism.

\section{REFERENCES}

[1] S. Alabady, et al., "Throughput and Delay Analysis of IEEE 802.11 DCF in the Presence of Hidden Nodes for Multi-Hop Wireless Networks," Wireless Personal Communications, Vol. 79, pp. 907-927, 2014.

[2] P. Mahasukhon, et al., "Performance Analysis of Multi-hop IEEE 802.11 DCF Backhaul Networks," International Confernce on Wireless and Mobile Computing on Networking and Communications (WiMOB), pp.69-74, 2008.

[3] A. Maher and Z. Khalaf, "Design and Performance Analysis of Biomedical Wireless Senser Network," M.Sc. Thesis, Northern Technical University, 2017.

[4] B. Maqhat, et al., "Performance Analysis of Fair Scheduler for A-MSDU Aggregation in IEEE802.11n Wireless Networks," 2014 2nd International Conference on Electrical, Electronics and System Engineering (ICEESE), 978-1-4799-7201-2/14/\$31.00@2014 IEEE.

[5] Y. Lin and V. W. S. Wong, "WSN01-1: Frame Aggregation and Optimal Frame Size Adaptation for IEEE 802.11n WLANs, " San Francisco, CA, USA, IEEE, 2006.

[6] D. Skordoulis, et al., "IEEE 802.11n MAC frame aggregation mechanisms for next-generation high-throughput WLANs," IEEE Wireless Communications, Vol. 15, Feb 2008.

[7] E. Perahia, "IEEE 802.11n Development: History, Process, and Technology," Communications Magazine, IEEE, Vol. 46, pp. 48-55, 2008.

[8] I. A. Alimi, et al., "A Hybrid Coding Technique for Efficient Bandwidth Usage in Conformity with IEEE 802.11 WLAN Standard," International Journal of Electrical and Computer Engineering (IJECE), Vol. 3, pp. 593-602, No. 5, Oct 2013

[9] A. Z. Younis, "Performance Analysis of IEEE 802.11ac Based WLAN in Wireless Communication Systems," International Journal of Electrical and Computer Engineering (IJECE), Vol. 9, pp. 1131-1136, No. 2, Apr 2019.

[10] Mir M. and Sauman B., "Performance Improvement of IEEE 802.11n WLANs via Frame Aggregation in NS-3," International Conference on Electrical, and Communication Engineering (ECCE), INSPEC NO. 16851147, Bangladesh, 2017.

[11] B. Abidi, et al., "Wireless Senser Networks in Biomedical: Wireless Body Area Networks," Europe and MENA Cooperation Advances in Information and Communication Technologies, Vol. 520, pp. 321-329, No. 33, 2017.

[12] D. Lee, et al., "Dynamic Traffic Prioritization and TXOP Allocation in 802.11e Based Multihop Wireless Networks," International Journal of Computer Science and Network Security (IJCSNS), Vol. 12, No. 2, Feb 2012.

[13] R. vohra, et al., "WLAN Performance Improvisation by Fine Tuning IEEE 802.11 Parameters," International Journal of Computer Applications (IJCA), Vol. 43, No. 6, April 2012.

[14] S. Q. Ameen and F. L. Khaleel, "Wireless Mesh Networks Based on MBPSO Algorithm to Improvement Throughput," International Journal of Electrical and Computer Engineering (IJECE), Vol. 8, pp. 4374-4381, No. 6, Dec 2018.

[15] P. Siripongwutikom, "Throughput Analysis of An IEEE 802.11b Multihop Ad hoc Network," In TENCON 2006. IEEE Region 10 Conference IEEE, pp.1-4, 2006.

[16] V. A. Siris, et al., "A simple End-to-end Throughput Model for 802.11Multi-ratio Multi-rate Wireless Mesh Networks," IEEE Communications Letters, Vol. 15, pp. 635-637, 2011.

[17] Z. k. Farej and M. M. Jasim, "Investigation on the Performance of the IEE802.1 In Based Wireless Networks for Multimedia Services," 2018 2nd International Conference for Engineering, Technology and Sciences of Al-Kitab (ICETS), added to IEEE Xplore: 30 May 2019.

[18] Y. Xiao, "IEEE 802.11n: Enhancements for higher throughput in wireless LANS," IEEE Wireless Communications, Vol. 12, Dec 2005.

[19] I. Paudel and D. Zeghlache, "QoS Provisioning in Future Wireless Local Area Networks," Ph.D. Thesis 2015TELE0004, Telecom SudParis, Evry, France, 15 Jan. 2015.

[20] M. I. Youssef, et al., "Performance Enhancement of MIMO-OFDM using Redundant Residue Number System," International Journal of Electrical and Computer Engineering (IJECE), Vol. 8, pp. 3902-3912, No. 5, Oct 2018.

[21] M. A. Al-Maqri, et al., "Review on QoS provisioning approaches for supporting video traffic in IEEE802.11e: challenges and issues," DOI 10.1109/ACCESS.2018.2872770, IEEE Access, 2018.

[22] A. Malik, et al., "QoS in IEEE 802.11-based Wireless Networks: A Contemporary Survey," Journal of Network and Computer Applications, DOI: 10.1016/j.jnca.2015.04.016, Vol. 55, pp. 24-46, Sep. 2015.

[23] M. Hameed and J. Jasperneite, "Performance Evaluation of IEEE 802.11e for Industrial Wireless Networks," M.Sc. Thesis 15181037, Ostwestfalen-Lippe University of Applied Sciences - Höxter, Lemgo, Germany, Aug. 2007.

[24] S. Xu, et al., "Layer-2 Multi-hop IEEE 802.11 Architecture: Design and Performance Analysis," IEEE Proc.Communication, Vol. 151, No. 5, Oct 2004.

[25] L. T. Nguyen, et al., "Performance Analysis of IEEE 802.11 in Multi-hop Wireless Networks," Proc. of the 3rd International Conference on Mobile Ad-hoc and Sensor Networks (MSN), Springer-Verlag Berlin, 2007.

[26] J. A. Mohamed, "Wireless Local Area Network WLAN 802.11," DIT, Digital Networks, ETU 08102, May 2016. 


\section{BIOGRAPHIES OF AUTHORS}

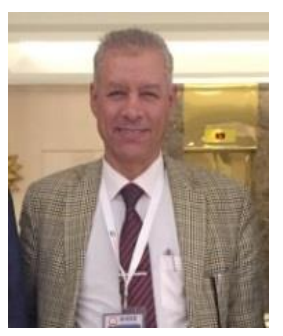

Dr. Ziyad Khalaf Farej is a lecturer and has BSc. in Electronic System Eng. from Cranfield University/UK with honor degree in 1989.MSc. in Spread Spectrum System/FH from Mosul University in 2003. Ph.D. in Computer and Communication Networks (Mesh Network based WIMAX IEEE 802.16 Standard technology) from Mosul University in 2012 with excellent grade. He has been to Salford Greater Manchester University/UK in Research Scholarship for 6 months during his Ph.D. studying period.

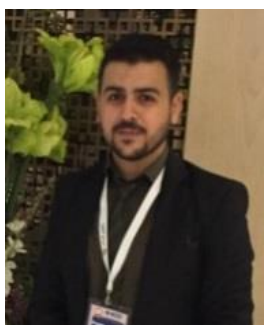

Mustafa Mohammad Jasim has BSc. in Computer Engineering from Technical Engineering Collage/Mosul in 2013, now he is a post-graduate student for the master degree in Computer Engineering (Wireless Networks Based on IEEE 802.11n Standard) at Technical College/Mosul. 